\title{
Subepithelial Spread of Early Gastric Signet Ring Cell Carcinoma: How Far They Can Reach?
}

\author{
Yong Moon Lee ${ }^{a}$ Sun Hyung Kang ${ }^{b}$ Ju Seok Kim ${ }^{b}$ Hyuk Soo Eun ${ }^{b}$ \\ Jong Seok Joo ${ }^{b}$ Woo Sun Rou ${ }^{b}$ Jae Ho Park ${ }^{b}$ Hee Seok Moon ${ }^{b}$ \\ Eaum Seok Lee $^{b}$ Seok Hyun Kim ${ }^{b}$ Jae kyu Sung ${ }^{b}$ Byung Seok Lee ${ }^{b}$ \\ Hyun Yong Jeong $^{b}$ Min Kyung Yeo ${ }^{c}$ Kyu Sang Song ${ }^{c}$ Hee Min Yoo ${ }^{d}$
}

aDepartment of Pathology, Dankook University School of Medicine, Cheonan, Republic of Korea; bivision of Gastroenterology and Hepatology, Department of Internal Medicine, Chungnam National University School of Medicine, Daejeon, Republic of Korea; 'Department of Pathology, Chungnam National University School of Medicine, Daejeon, Republic of Korea; ${ }^{d}$ Center for Bioanalysis, Korea Research Institute of Standards and Science, Daejeon, Republic of Korea

\section{Keywords}

Signet ring cell · Subepithelial spread · Endoscopic resection

\begin{abstract}
Introduction: Although signet ring cell carcinoma (SRC) is a poorly differentiated cancer subtype, recent studies suggest that endoscopic resection can be applied in small, mucosal early gastric SRC. However, other studies report frequent positive lines at the lateral resection margin after endoscopic treatment. Subepithelial spread beneath normal mucosa can exist in SRC, and such lesions may be the cause of positive margins after endoscopic resection. Thus, we conducted a retrospective study in order to evaluate the significance of subepithelial spread in early gastric SRC. Method: Medical records of early gastric SRC patients who underwent surgery or endoscopic resection from January 2011 to December
\end{abstract}

karger@karger.com www.karger.com/ddi

Karger $\stackrel{\text { ' }}{5}$

GOPEN ACCESS
(C) 2020 The Author(s)

Published by S. Karger AG, Basel

This article is licensed under the Creative Commons AttributionNonCommercial-NoDerivatives 4.0 International License (CC BY NC-ND) (http://www.karger.com/Services/OpenAccessLicense). Usage and distribution for commercial purposes as well as any distribution of modified material requires written permission.
2016 at a single tertiary hospital (Daejeon, South Korea) were reviewed to examine subepithelial spread and clinical datum. Two expert pathologists reviewed all pathologic specimens, and only patients showing a pure SRC component were included. Results: Eighty-six patients were initially enrolled, and subepithelial spread existed in 62 patients (72.1\%). The mean distance of subepithelial spread was $1,132.1 \mu \mathrm{m}$, and the maximal distance was $6,000 \mu \mathrm{m}$. Only discoloration was significantly associated with the presence of a subepithelial spread $\left(p<0.05, x^{2}\right.$ test, and logistic regression test). Distance of subepithelial spread did not correlate with total lesion size. Conclusion: Subepithelial spread of early gastric SRC occurs frequently and can reach up to 6 $\mathrm{mm}$. Lesion discoloration may be associated with the presence of subepithelial spread. Our results suggest that careful decision of the margin is needed when performing endoscopic resection of early gastric SRC.

(c) 2020 The Author(s)

Published by S. Karger AG, Basel

Dr. Sun Hyung Kang

Department of Internal Medicine, Chungnam National University

Munhwaro 282

Daejeon 35015 (Republic of Korea)

porrtos@hanmail.net 


\section{Introduction}

Endoscopic resection is now widely accepted as a primary treatment option in early gastric cancer (EGC), especially in Korea and Japan $[1,2]$. EGC is defined as a cancer limited to the mucosal or submucosal layer irrespective of lymph node (LN) status [3]. The treatment method, surgical or endoscopic resection, mainly depends on risk of LN metastasis [4]. Gotoda et al. [5] suggested low prevalence of LN metastasis in cases of shallow submucosal invasion, size $<3 \mathrm{~cm}$ without lymphatic or venous permeation, and mucosal cancer without ulceration irrespective of its size. However, the possibility of endoscopic resection in undifferentiated or poorly differentiated EGC is still under debate. While recent metaanalysis revealed that risk of LN metastasis was significantly higher in undifferentiated EGC treated under the expanded criteria of endoscopic submucosal dissection (ESD) when compared to EGC treated under the absolute criteria [6], other long-term follow-up studies showed feasible level of recurrence and survival data in undifferentiated EGC after ESD [7-9]. Although the complete resection rate is lower in undifferentiated EGC, only few local recurrence and distant metastasis were observed after achievement of curative resection [7-10].

Signet ring cell carcinoma (SRC) is a subtype of diffuse or undifferentiated cancer $[11,12]$. Unlike other poorly or undifferentiated cancers, early stage gastric SRC shows favorable prognosis. In a previous Korean study, SRC had lower LN metastasis and longer duration of survival than non-SRC [13]. Follow-up studies revealed that early gas- tric SRC smaller than $2 \mathrm{~cm}$ showed minimal risk of LN metastasis, suggesting that early gastric SRC may be a candidate for endoscopic resection [14-17]. However, the previous retrospective studies were based on pathologic results obtained after surgical resection. Early gastric SRC generally shows low curative resection after endoscopic resection due to lateral cut end-positivity [18]. Lateral cut end-positivity originates from the biologic behavior of SRC. Kim et al. [19] suggested two types of growth pattern in SRC: expansive and infiltrative type. Infiltrative type SRC is mostly located beneath normal appearing mucosa, and atrophy (with or without intestinal metaplasia) was the only risk factor for subepithelial (SE) spreading of SRC [19].

Unfortunately, there is no research regarding the distance of SE spreading in SRC. Assessing the degree of lateral spread in SRC could be of great value when deciding the lateral margin for endoscopic resection in SRC. Therefore, we aimed to study the maximal and average distance of lateral spreading in early gastric SRC. Percentage of SE spread and endoscopic, pathologic, and clinical factors related with SE spread were also evaluated.

\section{Material and Methods}

\section{Study Population}

We reviewed medical records and pathologic specimens of early gastric SRC patients who underwent surgery or endoscopic resection from January 2011 to December 2016 in Chungnam National University Hospital. All patients were diagnosed as pure SRC without other cell components. Patients who had mixed histology with a SRC component and other cell types were excluded
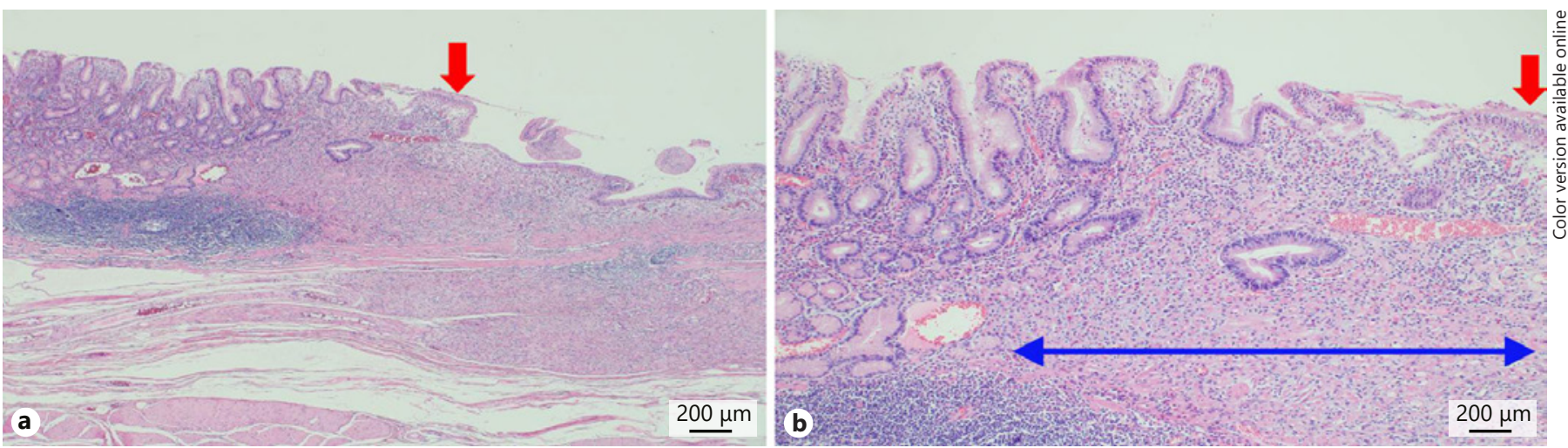

Fig. 1. Case of early gastric signet ring cell carcinoma showing perpendicular and horizontal spread. a The perpendicular spread makes submucosal invaded (pT1b), and typical mucosal erosion is terminated, which is the margin of the tumor detected by endoscopy (red arrow) (H\&E, $\times 40)$. b The horizontal spread of SRC through mucosa makes the difference (blue arrow; $1,500 \mu \mathrm{m}$ ) of the tumor border between microscopically and endoscopically (red arrow) determined $(\mathrm{H} \& \mathrm{E}, \times 100)$. 
Table 1. Patients characteristics

\begin{tabular}{ll}
\hline Patients characteristics & Patients, $n$ \\
\hline Gender (male:female) & $52: 34$ \\
Age (mean \pm SD) & $56.4 \pm 12.473$ \\
Depth of invasion (M cancer:SM cancer) & $69: 17$ \\
Lymphovascular invasion (no:yes) & $73: 13$ \\
Lymph node invasion (no:yes, only surgical cases) & $48: 3$ \\
Presence of subepithelial spread & $\mathbf{6 2 / 8 6 , 7 2 . 1 \%}$ \\
Atrophic gastrits (no:yes) & $9: 77$ \\
Helicobacter pylori (no:yes:unknown) & $40: 28: 18$ \\
Distance of subepithelial spread from main mass, mean (minimum to maximal value) $\mathbf{1 , 1 3 2} \boldsymbol{\mu m}(\mathbf{5 0 - 6 , 0 0 0} \boldsymbol{\mu m})$ \\
Endoscopic appearance (I:IIa:IIb:IIc:IIII) & $2: 2: 24: 42: 16$ \\
Location (cardia:UB:MB:LB:antrum:angle) & $0: 4: 5: 28: 33: 16$ \\
Color change (redness:discoloration:mixed) & $28: 23: 35$ \\
Treatment method (operation:ESD) & $51: 35$
\end{tabular}

SD, standard deviation; M, mucosal; SM, submucosal; UB, upper body; MB, mid-body; LB, lower body; ESD, endoscopic submucosal dissection.
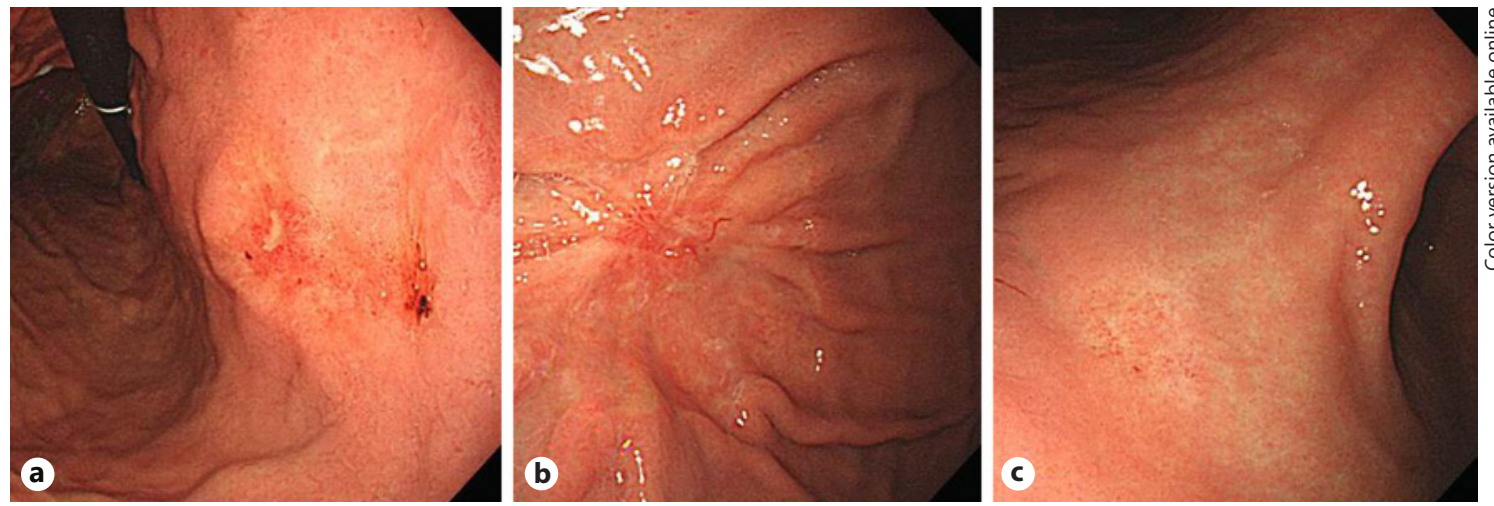

Fig. 2. Color changes of early gastric signet ring cell carcinoma: Redness (a), mixed (b), and discoloration (c).

because histologic heterogeneity might have influence on study data. A total of 86 patients were enrolled. Subtotal or total gastrectomy with D1 $+\alpha$ or D2 lymphadenectomy was performed, as well as ESD. ESD were performed by 4 expert endoscopists who performed $>5,000$ gastroscopies and 200 ESD procedures for EGC. Surgery was conducted by 2 expert surgeons with experience of performing $>1,000$ cases of gastrectomy.

\section{Pathologic Examination}

All pathologic specimens were reviewed by 2 expert pathologists (Y.M.L. and K.S.S.). Pathological specimens obtained after gastrectomy were sectioned at 4-mm intervals, and those obtained after ESD were sectioned at 3-mm intervals. Distance of SE spread was measured during pathologic review by calculating the distance from the terminal of the surface lesion (cancer) to the other terminal of SE spread (Fig. 1). Other pathologic data such as depth of invasion, lymphovascular invasion, LN metastasis, and presence of Helicobacter pylori were retrospectively evaluated with existing pathologic medical records.

\section{Endoscopic Examination}

All endoscopic procedures including ESD and preoperative gastroscopy were performed with a video endoscope (H240 and H260; Olympus Medical Systems, Tokyo, Japan), and chromoendoscopy with indigo-carmine was used occasionally. Endoscopic pictures were reviewed by 1 endoscopist (S.H.K.). Endoscopic review was performed with images saved in the picture archiving and communication system (PACS) of Chungnam National University Hospital. Endoscopic appearance, color change, and location were evaluated. Presence of atrophic gastritis was also evaluated. Color change was defined as redness (patch erythematous change), discoloration (disappearance of pinkish color of normal mucosa), and mixed patterns (mixture of erythematous change and discoloration) (Fig. 2).

\section{Statistical Methods}

Statistical analysis was performed using the SPSS software (version 18.0, Chicago, IL, USA). Univariate analysis using the $\chi^{2}$ test and multivariate logistic regression analysis were performed to 
Table 2. Distances of subepithelial spread of early gastric signet ring cell carcinoma from main mass

\begin{tabular}{lc}
\hline Distance from main mass & Patients, $n$ \\
\hline$<1,000 \mu \mathrm{m}$ & $41 / 62,66.1 \%$ \\
$1,000 \mu \mathrm{m} \leq$ to $<2,000 \mu \mathrm{m}$ & $4 / 62,6.5 \%$ \\
$2,000 \mu \mathrm{m} \leq$ to $<3,000 \mu \mathrm{m}$ & $9 / 62,14.5 \%$ \\
$3,000 \mu \mathrm{m} \leq$ & $8 / 62,12.9 \%$ \\
\hline
\end{tabular}

Table 4. Risk factor of subepithelial spread (multivariate analysis)

\begin{tabular}{llll}
\hline Color change & OR & $\beta$-coefficient & $p$ value \\
\hline Redness (reference) & & & \\
Discoloration & $4.117(1.112-15.246)$ & 1.415 & 0.034 \\
Mixed & 3.467 & 1.243 & 0.029
\end{tabular}

OR, odd ratio. Logistic regression test.

find risk factors of SE spreading in SRC. Pearson correlation was performed to evaluate relationship between superficial mass size and length of SE spreading. The accepted level of statistical significance was $p<0.05$.

\section{Results}

Medical records of 86 pure SRC patients were assessed. Number of male patients was 52, and mean age was 56.4 years. Fifty-one patients received gastrectomy, and ESD was performed in the 35 patients. Presence of SE spread was found in 62 patients $(72.1 \%)$, and the mean distance of SE spread was $1,132 \mu \mathrm{m}(50-6,000 \mu \mathrm{m})$ in 62 patients. The findings are listed in Table 1. Thirty-nine patients from the surgery group and 23 patients from the ESD group showed SE spread. There was no difference between the two groups in mean, median, minimum, and maximal value of SE spread. SE spread in 41 patients was $<1,000 \mu \mathrm{m}(66.1 \%)$. Eight patients showed SE spread over $3,000 \mu \mathrm{m}$ (12.9\%) (Table 2). Comparative analysis using the $\chi^{2}$ test was performed between the absence and presence of SE spread. There were no differences in sex, invasion depth, lymphovascular invasion, LN metastasis, atrophic gastritis, presence of Helicobacter pylori, size of lesion, and endoscopic appearance between the two groups. Only color change seen by the endoscopic image was significant ( $\chi^{2}$ test, $p=0.028$, Table 3 ). The presence of discoloration and mixed pattern of color change (discoloration + redness) significantly increased the risk of SE
Table 3. Risk factors of presence of subepithelial (SE) spread

\begin{tabular}{lccc}
\hline Risk factors & $\begin{array}{l}\text { Absence of } \\
\text { SE spread }\end{array}$ & $\begin{array}{l}\text { Presence of } \\
\text { SE spread }\end{array}$ & $\begin{array}{l}p \\
\text { value }\end{array}$ \\
& & & \\
Sex (male:female) & $13: 11$ & $39: 23$ & 0.472 \\
Depth (M cancer:SM cancer) & $21: 3$ & $48: 14$ & 0.231 \\
LVI (yes:no) & $1: 23$ & $12: 50$ & 0.069 \\
LN metastasis (yes:no) & $0: 12$ & $3: 36$ & 0.439 \\
Atrophic gastritis (yes:no) & $21: 3$ & $56: 6$ & 0.268 \\
Helicobacter pylori (yes:no) & $9: 10$ & $31: 18$ & 0.178 \\
Size of lesion & & & 0.412 \\
$\quad<1 \mathrm{~cm}$ & 10 & 20 & \\
$\quad 1 \mathrm{~cm} \leq$ to $<2 \mathrm{~cm}$ & 7 & 24 & \\
$\quad 2 \mathrm{~cm} \leq$ to $<3 \mathrm{~cm}$ & 6 & 10 & \\
$\quad 3 \mathrm{~cm} \leq$ & 1 & 8 & \\
Endoscopic appearance & & & 0.772 \\
$\quad$ I & 0 & 1 & \\
IIa & 1 & 1 & \\
$\quad$ IIb & 6 & 19 & \\
$\quad$ IIc & 14 & 28 & \\
III & 3 & 13 & \\
Color change & & & 0.028 \\
$\quad$ Redness & 13 & 15 & \\
$\quad$ Discoloration & 4 & 19 & \\
$\quad$ Mixed & 7 & 28 & \\
\hline
\end{tabular}

M, mucosal; SM, submucosal; LVI, lymphovascular invasion; LN, lymph node. $\chi^{2}$ test.

spread compared with redness (logistic regression test, $p<0.05$, Table 4). There was no correlation between pathologically measured size of lesion and length of SE spread (Spearman's rank correlation, $p=0.708, r=0.049$, Fig. 3).

\section{Discussion}

Despite the minimal risk of LN metastasis and good prognosis, ESD had not been accepted as a standard treatment in early gastric SRC. Unlike differentiated EGC, en bloc endoscopic resection could not confirm complete resection in early gastric SRC mainly due to the lateral margin [18]. However, long-term outcome of ESD in undifferentiated EGC showed acceptable level of recurrence in cases of curative resection $[10,18]$. Selection of eligible cases and achievement of sufficient safety lateral margin could make ESD an acceptable procedure in early gastric SRC.

The cause of lateral cut end-positivity in early gastric SRC originates from the biologic behavior of SRC [19]. The Infiltrative growth pattern of SRC gives rise to an un- 


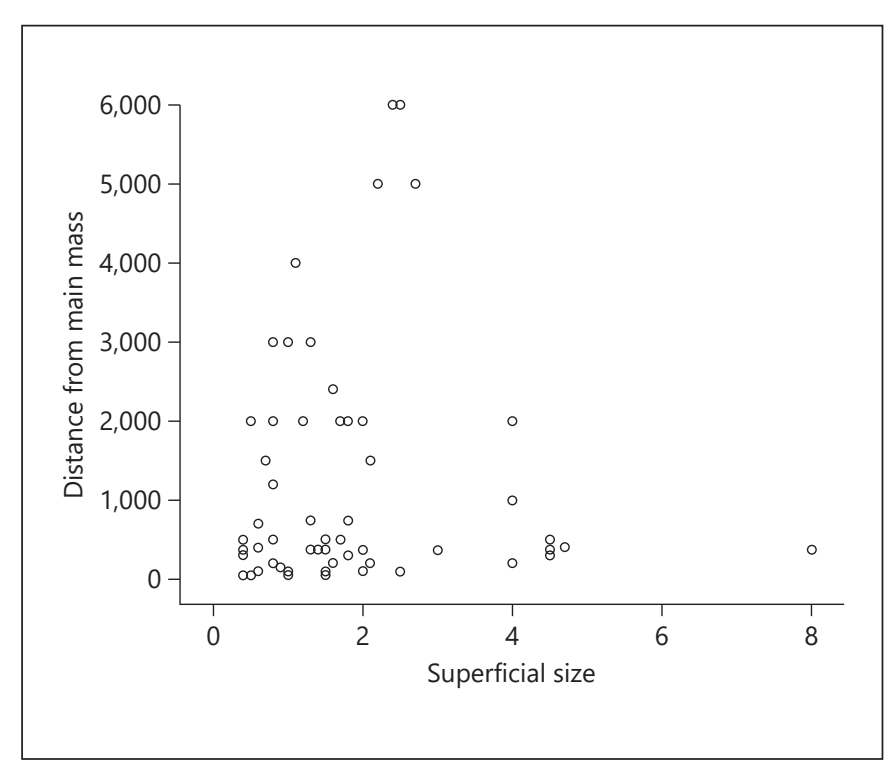

Fig. 3. Correlation between superficial size of lesion and distance of subepithelial spread. There was no definite correlation statistically (Pearson correlation).

detectable lesion beneath the normal mucosa. Physicians are unable to detect SE spread of SRC with endoscopy. Thus, it is very important to evaluate how far SE spreads. To our knowledge, this study is the first to evaluate the length of SE spread in early gastric SRC.

Our results show that SE spreading of early gastric SRC can reach up to $6,000 \mu \mathrm{m}$ from the terminal end of the surface lesion. A previous Korean study suggested that the safety margin $<3 \mathrm{~mm}$ was a risk factor of local recurrence [20]. Eight patients with SRC were enrolled in this study, and 6 patients (75\%) showed residual tumors. Another study showed that only $70 \%$ of early gastric SRC showed complete resection after endoscopic resection [18]. We presumed SE spread of SRC was the main cause of this phenomenon since $>70 \%$ of patients in our study showed SE spread.

In a previous Korean study, mucosal invasion pattern of early gastric SRC was classified into two types: infiltrative and expanding type [19]. Expanding type was defined as a tumor that had a margin that was clearly lined from nonneoplastic mucosa, whereas infiltrative type was defined as a tumor that showed diffuse spreading pattern. Infiltrative type showed higher prevalence of atrophic change and intestinal metaplasia than the expanding type. However, tumor color was not different [19]. In contrast to this previous study, our present results suggest that discoloration and mixed pattern of color change are

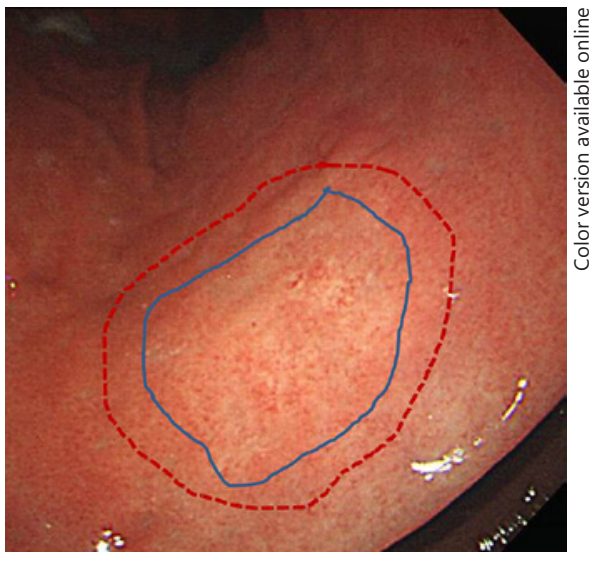

Fig. 4. Endoscopic feature of early gastric signet ring cell carcinoma. Gross margins are surrounded by the blue solid line, but the real range of cancer may reach up the red dotted line.

significantly related with SE spread, while atrophic change was not. The different results between studies may be due to the absence of histologic analysis for atrophy and intestinal metaplasia in the present study. We are also unable to provide a theoretical explanation between SE spread and tumor color. Compared to reddish lesion, risk of SE spread was increased 3-4 times in discoloration and mixed color. Our results suggest that endoscopists should consider the presence of SE spread when a discolored EGC lesion with signet ring cell histology is discovered. Endoscopists must also be aware that cancer cells might exist more widely than the visible range of the tumor (Fig. 4).

A different Korean study suggested tumor size as a significant factor for curative resection via ESD in early gastric SRC [21]. It was assumed that the larger the lesion size (early gastric SRC), the longer the SE spread. However, there was no correlation between main mass size and distance of SE spread. Thus, the margin of early gastric SRC should be determined at least $6 \mathrm{~mm}$ larger than the visible range irrespective of lesion size.

Former studies regarding the risk of LN metastasis in early gastric SRC insisted that early gastric SRC smaller than $2 \mathrm{~cm}$ could be safely treated with endoscopic resection [14-17]. However, all of these former studies evaluated postoperative pathologic specimens. Due to possibility of SE spread, discrepancy may exist between size estimation of preoperative endoscopy and postoperative pathologic evaluation. Thus, endoscopists should narrow down the size criteria for indication of endoscopic resection in early gastric SRC. 
To avoid the positive line of resection after ESD in undifferentiated cancer, 4 quadrant biopsy around the EGC before ESD may be helpful. SE spread should be considered also for determining the ESD margin also in this situation. If the cancer was found in blind biopsy at the outer margin of EGC, the real margin might be wider than expectation due to SE spread.

While our study is the first to estimate SE spread in early gastric SRC, our study has several important limitations. Our study was conducted with a relatively small sample size in a single center. If more cases were included, it is possible that the range of SE spread may differ. Also, the status of atrophic gastritis was only evaluated by endoscopy, not through a pathologic exam. Another limitation is that the presence of Helicobacter pylori was evaluated with a pathologic specimen after surgery or ESD. Routine exam with the urea breath test or rapid urease test was not performed. In addition, there were differences between surgical resection specimens and ESD specimens. Differences in the processing of pathologic specimens between surgical resection and ESD may have affected accurate assessment of SE spread. Moreover, SE spread was measured only parallel to the section of specimens.

In conclusion, SE spread of early gastric SRC was frequent and prominent in lesions with discoloration. Irrespective of lesion size, SE spread may exceed maximally $6 \mathrm{~mm}$ in our study. Our results suggest that careful decision of lateral resection margin is needed when performing endoscopic resection of early gastric SRC. A wider lateral margin is desirable in endoscopic resection of SRC than other histologic type of EGC.

\section{Acknowledgement}

There is no special acknowledgement in this article.

\section{Statement of Ethics}

All procedures followed were in accordance with the ethical standards of the responsible committee on human experimentation (institutional and national) and with the Helsinki Declaration of 1964 and later versions. This study was approved by the Institutional Review Board of Chungnam National University Hospital (IRB number; 2019-01-053), and written consent was waived because of the retrospective design of the study.

\section{Disclosure Statement}

The authors have no conflict of interest to declare.

\section{Funding Sources}

The authors have no sources of funding to declare.

\section{Author Contributions}

Conceptualization: S.H.K.; methodology: J.S.K. and H.S.E.; software: J.S.J. and W.S.R.; validation: Y.M.L. and S.H.K.; formal analysis: Y.M.L., J.H.P., and M.H.S.; investigation: Y.M.L., M.K.Y., K.S.S., and S.H.K.; resources: Y.M.L., Y.M.K., K.S.S., S.H.K., and J.H.Y.; data curation: Y.M.L., E.S.L., S.H.K., J.K.S., and B.S.L.; original draft preparation: Y.M.L. and H.M.Y.; review and editing: S.H.K.; visualization: J.S.K., J.S.J., and J.H.P.; supervision: S.H.K., B.S.L., and H.Y.J.; project administration: S.H.K.

\section{References}

1 Chung IK, Lee JH, Lee SH, Kim SJ, Cho JY, Cho WY, et al. Therapeutic outcomes in 1,000 cases of endoscopic submucosal dissection for early gastric neoplasms: Korean ESD Study Group multicenter study. Gastrointest Endosc. 2009;69(7):1228-35.

2 Oda I, Gotoda T, Hamanaka H, Eguchi T, Saito Y, Matsuda T, et al. Endoscopic submucosal dissection for early gastric cancer: technical feasibility, operation time and complications from a large consecutive series. Dig Endosc. 2005; 17:54-8.

3 Carter KJ, Schaffer HA, Ritchie WP Jr. Early gastric cancer. Ann Surg. 1984;199(5):604-9.

4 Ono H, Kondo H, Gotoda T, Shirao K, Yamaguchi H, Saito D, et al. Endoscopic mucosal resection for treatment of early gastric cancer. Gut. 2007;48(2):225-9.

Subepithelial Spread of Early Gastric SRC
5 Gotoda T, Yanagisawa A, Sasako M, Ono H, Nakanishi Y, Shimoda T, et al. Incidence of lymph node metastasis from early gastric cancer: estimation with a large number of cases at two large centers. Gastric Cancer. 2000; $3(4): 219-25$.

6 Abdelfatah MM, Barakat M, Lee H, Kim JJ, Uedo N, Grimm I, et al. The incidence of lymph node metastasis in early gastric cancer according to the expanded criteria in comparison with the absolute criteria of the Japanese Gastric Cancer Association: a systematic review of the literature and meta-analysis. Gastrointest Endosc. 2018;87:338-47.

7 Jeon HK, Lee SJ, Kim GH, Park DY, Lee BE, Song GA. Endoscopic submucosal dissection for undifferentiated-type early gastric cancer: short- and long-term outcomes. Surg Endosc. 2018;32:1963-70.
$8 \mathrm{Kim} \mathrm{JH}$, Kim YH, Jung DH, Jeon HH, Lee YC Lee H, et al. Follow-up outcomes of endoscopic resection for early gastric cancer with undifferentiated histology. Surg Endosc. 2014;28(9):2627-33.

9 Ahn JY, Jung HY, Choi KD, Choi JY, Kim MY, Lee JH, et al. Endoscopic and oncologic outcomes after endoscopic resection for early gastric cancer: 1,370 cases of absolute and extended indications. Gastrointest Endosc. 2011;74(3):485-93.

10 Abe S, Oda I, Suzuki H, Nonaka S, Yoshinaga $S$, Odagaki T, et al. Short- and long-term outcomes of endoscopic submucosal dissection for undifferentiated early gastric cancer. Endoscopy. 2013;45(9):703-7.

11 Sugano H, Nakamura K, Kato Y. Pathological studies of human gastric cancer. Acta Pathol Jpn. 1982;32(Suppl 2):329-47. 
12 Lauren P. The two histological main types of gastric carcinoma: diffuse and so-called intestinal-type carcinoma. an attempt at a histoclinical classification. Acta Pathol Microbiol Scand. 1965;64:31-49.

13 Hyung WJ, Noh SH, Lee JH, Huh JJ, Lah KH, Choi SH, et al. Early gastric carcinoma with signet ring cell histology. Cancer. 2002; 94(1):78-3.

14 Kang HJ, Kim DH, Jeon TY, Lee SH, Shin N, Chae SH, et al. Lymph node metastasis from intestinal-type early gastric cancer: experience in a single institution and reassessment of the extended criteria for endoscopic submucosal dissection. Gastrointest Endosc. 2010;72(3):508-15.
15 Lee SH, Jee SR, Kim JH, Seol SY. Intramucosal gastric cancer: the rate of lymph node metastasis in signet ring cell carcinoma is as low as that in well-differentiated adenocarcinoma. Eur J Gastroenterol Hepatol. 2015;27(2):1704.

16 Park JM, Kim SW, Nam KW, Cho YK, Lee IS, Choi MG, et al. Is it reasonable to treat early gastric cancer with signet ring cell histology by endoscopic resection? Analysis of factors related to lymph-node metastasis. Eur J Gastroenterol Hepatol. 2009;21(10):1132-5.

17 Kang SH, Kim JS, Moon HS, Lee ES, Kim SH, Sung JK, et al. Signet ring cell carcinoma of early gastric cancer, is endoscopic treatment really risky? Medicine. 2017;96(33):e7532.
18 Kim JH, Lee YC, Kim H, Song KH, Lee SK, Cheon JH, et al. Endoscopic resection for undifferentiated early gastric cancer. Gastrointest Endosc. 2009;69(4):e1-9.

19 Kim H, Kim JH, Lee YC, Kim H, Youn YH, Park $\mathrm{H}$, et al. Growth patterns of signet ring cell carcinoma of the stomach for endoscopic resection. Gut Liver. 2015;9(6):720-6.

20 Yun GW, Kim JH, Lee YC, Lee SK, Shin SK, Park JC, et al. What are the risk factors for residual tumor cells after endoscopic complete resection in gastric epithelial neoplasia? Surg Endosc. 2015;29(2):487-92.

21 Kim MN, Kim HK, Shim CN, Lee HJ, Lee H, Park JC, et al. Tumour size is related to the curability of signet ring cell early gastric cancer with endoscopic submucosal dissection: a retrospective single centre study. Dig Liver Dis. 2014;46(10):898-902. 